\title{
Infinitesimal Rigidity of Convex Polytopes *
}

\author{
C. Bauer \\ Zentrum Mathematik, Technische Universtät München, \\ D-80290 München, Germany \\ chbauer@mathematik.tu-muenchen.de
}

\begin{abstract}
Aleksandrov [1] proved that a simple convex $d$-dimensional polytope, $d \geq 3$, is infinitesimally rigid if the volumes of its facets satisfy a certain assumption of stationarity. We extend this result by proving that this assumption can be replaced by a stationarity assumption on the $k$-dimensional volumes of the polytope's $k$-dimensional faces, where $k \in\{1, \ldots, d-1\}$.
\end{abstract}

\section{Introduction and Main Result}

Let $P \subset \mathbb{R}^{d}$ be a polytope with interior points in $d$-dimensional Euclidean space $\mathbb{R}^{d}$, $d \geq 3$. In particular, $P$ belongs to the set $\mathcal{K}^{d}$ of convex bodies (nonempty, compact, convex sets) in $\mathbb{R}^{d}$. We equip $\mathbb{R}^{d}$ with the usual scalar product $\langle\cdot, \cdot\rangle$ and induced norm $\|\cdot\|$. We denote the unit sphere in $\mathbb{R}^{d}$ by $S^{d-1}$.

Let $F_{1}, \ldots, F_{n}$ be the facets, that is, the $(d-1)$-dimensional faces of $P$, and let $u_{1}, \ldots$, $u_{n} \in S^{d-1}$ be the corresponding outer unit normal vectors. If $h_{i}:=h\left(P, u_{i}\right)$ denotes the value of the support function of $P$ at $u_{i}$, then $P$ can be represented as

$$
P=\bigcap_{i=1}^{n} H^{-}\left(u_{i}, h_{i}\right),
$$

where $H^{-}\left(u_{i}, h_{i}\right):=\left\{x \in \mathbb{R}^{d} \mid\left\langle x, u_{i}\right\rangle \leq h_{i}\right\}$.

We now consider polytopes of the form

$$
P(x):=\bigcap_{i=1}^{n} H^{-}\left(u_{i}, x_{i}\right),
$$

\footnotetext{
* This work is part of the author's thesis [2] written at the Albert-Ludwigs-Universität, Freiburg im Breisgau, Germany.
} 
with $x=\left(x_{1}, \ldots, x_{n}\right) \in \mathbb{R}^{d}$. If $x$ lies in a sufficiently small neighborhood of $h:=$ $\left(h_{1}, \ldots, h_{n}\right)$, then $P(x)$ has the same number of facets as $P$. Furthermore, for each $i=1, \ldots, n$, the facet $F_{i}(x)$ of $P(x)$ is parallel to $F_{i}$.

In addition, we further assume that $P$ is a simple polytope, that is, each vertex of $P$ is contained in exactly $d$ facets of $P$. Then there is a neighborhood $U(h)$ of $h$ such that for all $x \in U(h)$ the polytopes $P(x)$ and $P$ are analogous (see Lemma 2.4 .12 of [8]). If the origin $o$ is an interior point of $P$, then $U(h)$ can be chosen such that

$$
\left.U(h)=\prod_{i=1}^{n}\right] h_{i}-\varepsilon, h_{i}+\varepsilon[\quad \text { with } \quad \varepsilon \in] 0, \min \left\{h_{1}, \ldots, h_{n}\right\}[\text {. }
$$

Hence, $o$ is also an interior point of $P(x)$ for all $x \in U(h)$.

The term analogous means that for an arbitrary $u \in S^{d-1}$ the intersection of $P$ with its supporting hyperplane in direction $u$ has the same dimension as the intersection of $P(x)$ with its supporting hyperplane in the same direction. Furthermore, these faces are parallel. If $F$ is a face of $P$ we denote by $F(x)$ the corresponding face of $P(x)$. We write $\mathcal{F}_{i}(P)$ for the set of $i$-dimensional faces of $P$, where $i \in\{0, \ldots, d-1\}$.

If $c=\left(c_{1}, \ldots, c_{d}\right) \in \mathbb{R}^{d}$ is fixed, then the above shows that for all $t \in \mathbb{R}$ satisfying $\max \left\{\left|t c_{1}\right|, \ldots,\left|t c_{n}\right|\right\}<\varepsilon$ the polytope $P(h+t c)$ is analogous to $P$ and hence has facets $F_{i}(c+t h), i=1, \ldots, n$. If the $(d-1)$-dimensional volume $V_{d-1}$ of each facet (considered as a function of $t$ ) satisfies the equation

$$
\left.\frac{d}{d t}\right|_{t=0} V_{d-1}\left(F_{i}(h+t c)\right)=0
$$

then $c=\left(\left\langle a, u_{1}\right\rangle, \ldots,\left\langle a, u_{n}\right\rangle\right)$ with $a \in \mathbb{R}^{d}$. This was proved by Aleksandrov [1, pp. 407-411]. We then say that $c$ is the support vector of a point. Aleksandrov's result shows that

$$
P(h+t c)=\bigcap_{i=1}^{n} H^{-}\left(u_{i}, h_{i}+t\left\langle a, u_{i}\right\rangle\right)=P+t a .
$$

Consequently, $P(h+t c)$ and $P$ are equal up to translation.

For $t$ with $\max \left\{\left|t c_{1}\right|, \ldots,\left|t c_{n}\right|\right\}<\varepsilon$ the polytope $P(h+t c)$ may be viewed as a deformation of the polytope $P$. Thus, Aleksandrov has proved that a simple polytope satisfying (2) is infinitesimally rigid. This means that the deformation is a translation if the derivatives of the facets' volumes are vanishing at the beginning of the deformation (that is, at time $t=0$ ).

In this work we generalize Aleksandrov's result. It was asked by Rolf Schneider (personal communication) whether in (2) one may replace the facets and $(d-1)$ dimensional volume, respectively, by the $k$-faces and $k$-dimensional volume $V_{k}$ for some fixed $k \in\{1, \ldots, d-1\}$. That this may be done is proved by the following theorem.

Theorem 1. Let $P \in \mathcal{K}^{d}, d \geq 3$, be a simple d-dimensional polytope. Let $u_{1}, \ldots, u_{n} \in$ $S^{d-1}$ be the pairwise disjoint outer normal vectors of $P$. Define the vector $h \in \mathbb{R}^{n}$ by $h:=\left(h\left(P, u_{1}\right), \ldots, h\left(P, u_{n}\right)\right)$. For $k \in\{1, \ldots, d-1\}$ and $c=\left(c_{1}, \ldots, c_{n}\right) \in \mathbb{R}^{n}$ 
assume that

$$
\left.\frac{d}{d t}\right|_{t=0} V_{k}(F(h+t c))=0
$$

for all $F \in \mathcal{F}_{k}(P)$. Then $c=\left(\left\langle a, u_{1}\right\rangle, \ldots,\left\langle a, u_{n}\right\rangle\right)$ with $a \in \mathbb{R}^{d}$.

The proof of this result requires several preliminaries.

In Section 2 we investigate the volume of $P(x)$ as a function of $x \in U(h)$. Here, we study the relationship of the $k$-fold partial derivatives of the volume and the $k$-dimensional volumes of the $k$-faces of $P(x)$.

In Section 3 we relate the volume of $P(x)$ to the notion of $k$-stress which was introduced by Lee [5].

Section 4 is devoted to the proof of Theorem 1.

\section{Simple Polytopes and Volume}

In what follows, we, without loss of generality, always assume that $o$ is an interior point of $P$ and $U(h)$ is as in (1). For $x \in U(h)$ let $V(x):=V_{d}(P(x))$ be the $d$-dimensional volume of $P(x)$. It is proved in [8, Lemma 5.1.2], that $V$ is a polynomial in $x$ which is homogeneous of degree $d$. Consequently, for $k \in\{1, \ldots, d\}$ and $i_{1}, \ldots, i_{k} \in\{1, \ldots, n\}$, the partial derivatives

$$
\frac{\partial^{k} V}{\partial x_{i_{k}} \cdots \partial x_{i_{1}}}(x)
$$

exist for all $x \in U(h)$.

The fact that the polytope $P$ is simple implies that for each $(d-k)$-dimensional face $F \in \mathcal{F}_{d-k}(P)$ there are exactly $k$ facets $F_{i_{1}}, \ldots, F_{i_{k}}$ of $P$ such that $F=F_{i_{1}} \cap \cdots \cap F_{i_{k}}$ (see Theorem 12.14 of [3]). The outer normal vectors $u_{i_{1}}, \ldots, u_{i_{k}}$ belonging to these facets are linearly independent. Furthermore, for all $x \in U(h)$, the face $F(x)$ has the representation $F(x)=F_{i_{1}}(x) \cap \cdots \cap F_{i_{k}}(x)$.

Our first objective is to clarify the connection between the $(d-k)$-dimensional volume $V_{d-k}(F(x))$ of $F(x)$ and the partial derivative $\left(\partial^{k} V / \partial x_{i_{k}} \cdots \partial x_{i_{1}}\right)(x)$. This is done in Theorem 4 . In order to prove this theorem we need some preliminaries. In what follows, we set $H(u, \tau):=\left\{x \in \mathbb{R}^{d} \mid\langle x, u\rangle=\tau\right\}$.

Lemma 2. Let $K \in \mathcal{K}^{d}$ be a convex body of dimension $\geq 1$. Let $u \in S^{d-1}$. We assume that $u$ is not orthogonal to the linear subspace which is parallel to aff $K$, the affine hull of $K$. Let $\left(\tau_{i}\right)_{i \in \mathbb{N}}$ be a sequence of real numbers converging to $\tau \in \mathbb{R}$ and satisfying $K \cap H\left(u, \tau_{i}\right) \neq \emptyset$ for all $i \in \mathbb{N}$. Then $\lim K \cap H\left(u, \tau_{i}\right)=K \cap H(u, \tau)$ in the Hausdorff metric.

Proof. For the proof we use Theorem 1.8.7 of [8].

Clearly, each limit of a sequence $\left(x_{i_{j}}\right)_{j \in \mathbb{N}}$ in $\mathbb{R}^{d}$ with $x_{i_{j}} \in K \cap H\left(u, \tau_{i_{j}}\right)$ for all $j \in \mathbb{N}$ belongs to $K \cap H(u, \tau)$. 
Now let $x \in K \cap H(u, \tau)$. We want to show that there is a sequence $\left(x_{i}\right)_{i \in \mathbb{N}}$ satisfying $x_{i} \in K \cap H\left(u, \tau_{i}\right)$ for all $i \in \mathbb{N}$ and $\lim x_{i}=x$.

We first assume that the hyperplane $H(u, \tau)$ supports $K$. Let $z \in$ relint $K$, where relint denotes the relative interior. Then, by elementary convexity,

$$
(\operatorname{conv}\{z, x\}) \backslash\{x\} \subset \text { relint } K .
$$

For $i \in \mathbb{N}$ define

$$
\alpha_{i}:=\frac{\tau_{i}-\tau}{\langle z, u\rangle-\tau} .
$$

We have $\alpha_{i} \geq 0$ since $H(u, \tau)$ supports $K$. Because $\left(\alpha_{i}\right)_{i \in \mathbb{N}}$ converges to 0 , the relation $\alpha_{i}<1$ holds if $i$ is sufficiently large. For $i \in \mathbb{N}$ with this property we define $x_{i}:=$ $\left(1-\alpha_{i}\right) x+\alpha_{i} z$. Then $x_{i} \in K$ because of (4). Furthermore, $\left\langle x_{i}, u\right\rangle=\tau_{i}$ and thus $x_{i} \in K \cap H\left(u, \tau_{i}\right)$. From $\lim \alpha_{i}=0$ we deduce $\lim x_{i}=x$.

If $H(u, \tau)$ is not a supporting hyperplane of $K$, then (relint $K) \cap H(u, \tau) \neq \emptyset$. We then define $K^{ \pm}:=K \cap H^{ \pm}(u, \tau)$. The convex bodies $K^{+}$and $K^{-}$have the same dimension as $K$ and $x \in K^{ \pm} \cap H(u, \tau)$. Furthermore, the hyperplane $H(u, \tau)$ supports $K^{+}$as well as $K^{-}$. For $\tau_{i} \neq \tau$ the hyperplane $H\left(u, \tau_{i}\right)$ intersects either $K^{+}$or $K^{-}$ but not both. As before, one can construct a sequence $\left(x_{i}\right)_{i \in \mathbb{N}}$ such that $\lim x_{i}=x$ and $x_{i} \in K \cap H\left(u, \tau_{i}\right)$ for all $i \in \mathbb{N}$.

Lemma 3. Let $k \in\{1, \ldots, d-1\}$ and let $u_{1}, \ldots, u_{k}, u \in S^{d-1}$, be linearly independent. Let $K \in \mathcal{K}^{d}$ be a convex body with interior points. If $d_{k}(u)$ denotes the distance from $u$ to $\operatorname{lin}\left\{u_{1}, \ldots, u_{k}\right\}$, then

$$
\begin{aligned}
V_{d-k}\left(K \cap H\left(u_{1}, \tau_{1}\right) \cap \cdots \cap H\left(u_{k}, \tau_{k}\right)\right) \\
\quad=\frac{1}{d_{k}(u)} \int_{-h(K,-u)}^{h(K, u)} V_{d-k-1}\left(K \cap H\left(u_{1}, \tau_{1}\right) \cap \cdots \cap H\left(u_{k}, \tau_{k}\right) \cap H(u, \tau)\right) d \tau
\end{aligned}
$$

for all $\tau_{1}, \ldots, \tau_{k} \in \mathbb{R}$.

Proof. Let $K^{\prime}:=K \cap H\left(u_{1}, \tau_{1}\right) \cap \cdots \cap H\left(u_{k}, \tau_{k}\right)$ be of dimension $d-k$ (otherwise, there is nothing to prove). Denoting by $\perp$ the orthogonal complement, aff $K^{\prime}$ has the form aff $K^{\prime}=\operatorname{lin}\left\{u_{1}, \ldots, u_{k}\right\}^{\perp}+x_{0}$ with $x_{0} \in \operatorname{lin}\left\{u_{1}, \ldots, u_{k}\right\}$. Let $u^{\prime}$ be the orthogonal projection of $u$ onto $\operatorname{lin}\left\{u_{1}, \ldots, u_{k}\right\}^{\perp}$.

We apply Fubini's theorem in the linear subspace $\operatorname{lin}\left\{u_{1}, \ldots, u_{k}\right\}^{\perp}$ which is parallel to aff $K^{\prime}$. Furthermore, we notice that $h\left(K^{\prime}-x_{0}, u^{\prime}\right)=h\left(K^{\prime}-x_{0}, u\right)$. We obtain

$$
\begin{aligned}
V_{d-k}\left(K^{\prime}\right) & =V_{d-k}\left(K^{\prime}-x_{0}\right) \\
& =\int_{-h\left(K^{\prime}-x_{0},-u^{\prime} /\left\|u^{\prime}\right\|\right)}^{h\left(K^{\prime}-x_{0}, u^{\prime} /\left\|u^{\prime}\right\|\right)} V_{d-k-1}\left(\left(K^{\prime}-x_{0}\right) \cap H\left(u^{\prime} /\left\|u^{\prime}\right\|, \tau\right)\right) d \tau \\
& =\frac{1}{\left\|u^{\prime}\right\|} \int_{-h\left(K^{\prime}-x_{0},-u^{\prime}\right)}^{h\left(K^{\prime}-x_{0}, u^{\prime}\right)} V_{d-k-1}\left(\left(K^{\prime}-x_{0}\right) \cap H\left(u^{\prime}, \tau\right)\right) d \tau \\
& =\frac{1}{\left\|u^{\prime}\right\|} \int_{-h\left(K^{\prime},-u\right)-\left\langle x_{0}, u\right\rangle}^{h\left(K^{\prime}, u\right)-\left\langle x_{0}, u\right\rangle} V_{d-k-1}\left(K^{\prime} \cap H\left(u, \tau+\left\langle x_{0}, u\right\rangle\right)\right) d \tau
\end{aligned}
$$




$$
\begin{aligned}
& =\frac{1}{\left\|u^{\prime}\right\|} \int_{-h\left(K^{\prime},-u\right)}^{h\left(K^{\prime}, u\right)} V_{d-k-1}\left(K^{\prime} \cap H(u, \tau)\right) d \tau \\
& =\frac{1}{\left\|u^{\prime}\right\|} \int_{-h(K,-u)}^{h(K, u)} V_{d-k-1}\left(K^{\prime} \cap H(u, \tau)\right) d \tau .
\end{aligned}
$$

Here, the last step follows because $K^{\prime} \subset K$. Since $\left\|u^{\prime}\right\|=d_{k}(u)$, the assertion follows.

The connection between the $(d-k)$-dimensional volume of $F_{i_{1}}(x) \cap \cdots \cap F_{i_{k}}(x)$ and the partial derivative $\left(\partial^{k} V / \partial x_{i_{k}} \cdots \partial x_{i_{1}}\right)(x)$ is given by the following theorem.

Theorem 4. Let $k \in\{1, \ldots, d\}$ and let numbers $i_{1}, \ldots, i_{k} \in\{1, \ldots, n\}$ be chosen such that $F_{i_{1}} \cap \cdots \cap F_{i_{k}} \in \mathcal{F}_{d-k}(P)$. Then

$$
\frac{\partial^{k} V}{\partial x_{i_{k}} \cdots \partial x_{i_{1}}}(x)=\left[u_{i_{1}}, \ldots, u_{i_{k}}\right]^{-1} V_{d-k}\left(F_{i_{1}}(x) \cap \cdots \cap F_{i_{k}}(x)\right)
$$

for all $x \in U(h)$. Here, $\left[u_{i_{1}}, \ldots, u_{i_{k}}\right]$ denotes the $k$-dimensional volume of the parallelepiped spanned by $u_{i_{1}}, \ldots, u_{i_{k}}$.

Proof. The assertion is proved by induction on $k$. Let $i \in\{1, \ldots, n\}$ and $a \in U(h)$. For $\left.x \in] h_{i}-\varepsilon, a_{i}\right]$ let

$$
P_{x}:=P\left(a_{1}, \ldots, a_{i-1}, x, a_{i+1}, \ldots, a_{n}\right) .
$$

From $o \in$ int $P_{x}$ and $x>0$, we deduce that $h\left(P_{x},-u_{i}\right)=h\left(P(a),-u_{i}\right)$. Hence,

$$
\begin{aligned}
V\left(a_{1}, \ldots,\right. & \left.a_{i-1}, x, a_{i+1}, \ldots, a_{n}\right) \\
& =\int_{-h\left(P_{x},-u_{i}\right)}^{h\left(P_{x}, u_{i}\right)} V_{d-1}\left(P_{x} \cap H\left(u_{i}, \tau\right)\right) d \tau \\
& =\int_{-h\left(P(a),-u_{i}\right)}^{x} V_{d-1}\left(P(a) \cap H^{-}\left(u_{i}, x\right) \cap H\left(u_{i}, \tau\right)\right) d \tau \\
& =\int_{-h\left(P(a),-u_{i}\right)}^{x} V_{d-1}\left(P(a) \cap H\left(u_{i}, \tau\right)\right) d \tau .
\end{aligned}
$$

From Lemma 2 we obtain that the function $\tau \mapsto V_{d-1}\left(P(a) \cap H\left(u_{i}, \tau\right)\right)$ is continuous on $\left.]-h\left(P(a),-u_{i}\right), a_{i}\right]$. Thus,

$$
\begin{aligned}
\frac{\partial V}{\partial x_{i}}(a) & =V_{d-1}\left(P(a) \cap H\left(u_{i}, a_{i}\right)\right) \\
& =V_{d-1}\left(F_{i}(a)\right) .
\end{aligned}
$$

Let $k \in\{2, \ldots, d\}$ and assume the assertion to be true for $k-1$. Let $i_{1}, \ldots, i_{k} \in$ $\{1, \ldots, n\}$ and $F_{i_{1}} \cap \cdots \cap F_{i_{k}} \in \mathcal{F}_{d-k}(P)$. After a suitable renumbering we may assume 
that $\left\{i_{1}, \ldots, i_{k}\right\}=\{1, \ldots, k\}$. The assumption of induction implies

$$
\frac{\partial^{k-1} V}{\partial x_{k-1} \cdots \partial x_{1}}(x)=\left[u_{1}, \ldots, u_{k-1}\right]^{-1} V_{d-k+1}\left(F_{1}(x) \cap \cdots \cap F_{k-1}(x)\right)
$$

for all $x \in U(h)$.

Let $a=\left(a_{1}, \ldots, a_{n}\right) \in U(h)$ and $\left.\left.x \in\right] h_{k}-\varepsilon, a_{k}\right]$. Define

$$
a_{x}:=\left(a_{1}, \ldots, a_{k-1}, x, a_{k+1}, \ldots, a_{n}\right)
$$

and $P_{x}:=P\left(a_{x}\right)$. If $d_{k-1}\left(u_{k}\right)$ denotes the distance from $u_{k}$ to $\operatorname{lin}\left\{u_{1}, \ldots, u_{k-1}\right\}$, then Lemma 3 implies

$$
\begin{aligned}
d_{k-1}\left(u_{k}\right) & V_{d-k+1}\left(F_{1}\left(a_{x}\right) \cap \cdots \cap F_{k-1}\left(a_{x}\right)\right) \\
& =d_{k-1}\left(u_{k}\right) V_{d-k+1}\left(P_{x} \cap H\left(u_{1}, a_{1}\right) \cap \cdots \cap H\left(u_{k-1}, a_{k-1}\right)\right) \\
& =\int_{-h\left(P_{x},-u_{k}\right)}^{h\left(P_{x}, u_{k}\right)} V_{d-k}\left(P_{x} \cap H\left(u_{1}, a_{1}\right) \cap \cdots \cap H\left(u_{k-1}, a_{k-1}\right) \cap H\left(u_{k}, \tau\right)\right) d \tau \\
& =\int_{-h\left(P(a),-u_{k}\right)}^{x} V_{d-k}\left(P_{x} \cap H\left(u_{1}, a_{1}\right) \cap \cdots \cap H\left(u_{k-1}, a_{k-1}\right) \cap H\left(u_{k}, \tau\right)\right) d \tau .
\end{aligned}
$$

Hence by (6),

$$
\begin{aligned}
{\left[u_{1}, \ldots, u_{k}\right] } & \frac{\partial^{k-1} V}{\partial x_{k-1} \cdots \partial x_{1}}\left(a_{x}\right) \\
= & d_{k-1}\left(u_{k}\right)\left[u_{1}, \ldots, u_{k-1}\right] \frac{\partial^{k-1} V}{\partial x_{k-1} \cdots \partial x_{1}}\left(a_{x}\right) \\
= & d_{k-1}\left(u_{k}\right) V_{d-k+1}\left(F_{1}\left(a_{x}\right) \cap \cdots \cap F_{k-1}\left(a_{x}\right)\right) \\
= & \int_{-h\left(P(a),-u_{k}\right)}^{x} V_{d-k}\left(P_{x} \cap H\left(u_{1}, a_{1}\right) \cap \cdots \cap H\left(u_{k-1}, a_{k-1}\right) \cap H\left(u_{k}, \tau\right)\right) d \tau \\
= & \int_{-h\left(P(a),-u_{k}\right)}^{x} V_{d-k}\left(P(a) \cap H\left(u_{1}, a_{1}\right) \cap \cdots \cap H\left(u_{k-1}, a_{k-1}\right) \cap H\left(u_{k}, \tau\right)\right) d \tau \\
= & \int_{-h\left(P(a),-u_{k}\right)}^{h_{k}-\varepsilon} V_{d-k}\left(P(a) \cap H\left(u_{1}, a_{1}\right) \cap \cdots \cap H\left(u_{k-1}, a_{k-1}\right) \cap H\left(u_{k}, \tau\right)\right) d \tau \\
& +\int_{h_{k}-\varepsilon}^{x} V_{d-k}\left(P(a) \cap H\left(u_{1}, a_{1}\right) \cap \cdots \cap H\left(u_{k-1}, a_{k-1}\right) \cap H\left(u_{k}, \tau\right)\right) d \tau .
\end{aligned}
$$

By Lemma 2, the function

$$
\tau \mapsto V_{d-k}\left(P(a) \cap H\left(u_{1}, a_{1}\right) \cap \cdots \cap H\left(u_{k-1}, a_{k-1}\right) \cap H\left(u_{k}, \tau\right)\right)
$$

is continuous on $\left.] h_{k}-\varepsilon, a_{k}\right]$. This follows, since for $\left.\left.\tau \in\right] h_{k}-\varepsilon, a_{k}\right]$ the intersection

$$
P(a) \cap H\left(u_{1}, a_{1}\right) \cap \cdots \cap H\left(u_{k-1}, a_{k-1}\right) \cap H\left(u_{k}, \tau\right)
$$


is the face of $P\left(a_{1}, \ldots, a_{k-1}, \tau, a_{k+1}, \ldots, a_{n}\right)$ corresponding to the face $F_{1} \cap \cdots \cap F_{k}$ of $P$. Consequently,

$$
\begin{aligned}
{\left[u_{1}, \ldots, u_{k}\right] } & \frac{\partial^{k} V}{\partial x_{k} \cdots \partial x_{1}}(a) \\
= & V_{d-k}\left(P(a) \cap H\left(u_{1}, a_{1}\right) \cap \cdots \cap H\left(u_{k-1}, a_{k-1}\right) \cap H\left(u_{k}, a_{k}\right)\right) \\
& =V_{d-k}\left(F_{1}(a) \cap \cdots \cap F_{k}(a)\right) .
\end{aligned}
$$

This proves the assertion.

Theorem 4 yields:

Proposition 5. Let $k \in\{1, \ldots, d\}$. Let $i_{1}, \ldots, i_{k} \in\{1, \ldots, n\}$ be such that $F_{i_{1}} \cap \cdots \cap$ $F_{i_{k}}=\emptyset$. Then

$$
\frac{\partial^{k} V}{\partial x_{i_{k}} \cdots \partial x_{i_{1}}}(x)=0
$$

for all $x \in U(h)$.

Proof. It suffices to prove the assertion for pairwise disjoint numbers $i_{1}, \ldots, i_{k} \in$ $\{1, \ldots, n\}$. Without loss of generality we assume that $\left\{i_{1}, \ldots, i_{k}\right\}=\{1, \ldots, k\}$. There exists $s \in\{1, \ldots, k-1\}$ such that $F_{1} \cap \cdots \cap F_{s} \in \mathcal{F}_{d-s}(P)$ after a suitable renumbering. We further assume that $s$ is the greatest number with this property. This means that each intersection of $s+1$ facets of $P$ with outer normal vectors among $\left\{u_{1}, \ldots, u_{k}\right\}$ is empty. Theorem 4 yields

$$
\frac{\partial^{s} V}{\partial x_{s} \cdots \partial x_{1}}(x)=\left[u_{1}, \ldots, u_{s}\right]^{-1} V_{d-s}\left(F_{1}(x) \cap \cdots \cap F_{s}(x)\right)
$$

for all $x \in U(h)$. Let $a \in U(h)$ and define $a_{x}:=\left(a_{1}, \ldots, a_{s}, x, a_{s+2}, \ldots, a_{n}\right)$, where $\left.x \in] h_{s+1}-\varepsilon, a_{s+1}\right]$. The choice of $s$ implies that $F_{1}(a) \cap \cdots \cap F_{s+1}(a)=\emptyset$. Thus,

$$
\begin{aligned}
F_{1}\left(a_{x}\right) \cap \cdots \cap F_{s}\left(a_{x}\right) & =P\left(a_{x}\right) \cap H\left(u_{1}, a_{1}\right) \cap \cdots \cap H\left(u_{s}, a_{s}\right) \\
& =P(a) \cap H\left(u_{1}, a_{1}\right) \cap \cdots \cap H\left(u_{s}, a_{s}\right) \cap H^{-}\left(u_{s+1}, x\right) \\
& =F_{1}(a) \cap \cdots \cap F_{s}(a) \cap H^{-}\left(u_{s+1}, x\right) \\
& =F_{1}(a) \cap \cdots \cap F_{s}(a),
\end{aligned}
$$

if $x$ lies in a sufficiently small leftside neighborhood of $a_{s+1}$. From (7) we deduce

$$
\frac{\partial^{s} V}{\partial x_{s} \cdots \partial x_{1}}\left(a_{x}\right)=\frac{\partial^{s} V}{\partial x_{s} \cdots \partial x_{1}}(a) \text {. }
$$

Hence,

$$
\frac{\partial^{s+1} V}{\partial x_{s+1} \cdots \partial x_{1}}(a)=0 .
$$

The assertion follows, since $s \leq k-1$ and $a \in U(h)$ was chosen arbitrarily. 


\section{Simple Polytopes and Stress}

The main tool for proving the extension of Aleksandrov's result is the notion of $k$-stress which was introduced by Lee [5] (see also [6] and [7]). The definition of this notion requires some more nomenclature.

As before, $P$ is a simple $d$-dimensional polytope which contains $o$ in its interior. The polar body of $P$ defines an abstract simplicial complex $\Delta$ on $\{1, \ldots, n\}$. For $k \in$ $\{1, \ldots, d-1\}$ set

$$
\Delta_{k}:=\left\{\left\{i_{1}, \ldots, i_{k}\right\} \subset\{1, \ldots, n\} \mid F_{i_{1}} \cap \cdots \cap F_{i_{k}} \in \mathcal{F}_{d-k}(P)\right\}
$$

Let

$$
\mathbb{Z}_{+}^{n}:=\left\{\left(r_{1}, \ldots, r_{n}\right) \in \mathbb{Z}^{n} \mid r_{i} \geq 0 \text { for all } i=1, \ldots, n\right\}
$$

For $r=\left(r_{1}, \ldots, r_{n}\right) \in \mathbb{Z}_{+}^{n}$ define

$$
x^{r}:=x_{1}^{r_{1}} \cdots x_{n}^{r_{n}} \text { and } \operatorname{supp} x^{r}:=\left\{i \in\{1, \ldots, n\} \mid r_{i} \neq 0\right\} .
$$

Furthermore, we set

$$
r !:=r_{1} ! \cdots r_{n} ! \quad \text { and } \quad|r|:=r_{1}+\cdots+r_{n}
$$

A linear $k$-stress (with respect to $u_{1}, \ldots, u_{n}$ ), where $k \in \mathbb{N} \cup\{0\}$, is a polynomial $b$ defined on $U(h)$ of the form

$$
b(x)=\sum_{\substack{r \in \mathbb{Z}_{+}^{n} \\|r|=k}} b_{r} \frac{x^{r}}{r !}
$$

satisfying

$$
b_{r}=0, \quad \text { if } \quad \operatorname{supp} x^{r} \notin \Delta,
$$

and

$$
\sum_{i=1}^{n} b_{s+e_{i}} u_{i}=o \quad \text { for all } \quad s \in \mathbb{Z}_{+}^{n} \quad \text { with } \quad|s|=k-1 .
$$

Here, $e_{1}, \ldots, e_{n}$ denote the canonical unit vectors of $\mathbb{R}^{n}$. The set of all linear $k$-stresses forms a vector space denoted by $S_{k}^{l}$.

For $k \in\{2, \ldots, d\}$ let $L_{k}\left(\mathbb{R}^{n} \times \cdots \times \mathbb{R}^{n} ; \mathbb{R}\right)$ be the vector space of multilinear real-valued functions defined on the $k$-fold cartesian product of $\mathbb{R}^{n}$. We define

$$
T_{k}: S_{k}^{l} \rightarrow L_{k}\left(\mathbb{R}^{n} \times \cdots \times \mathbb{R}^{n} ; \mathbb{R}\right)
$$


by $T_{k}(b)\left(e_{i_{1}}, \ldots, e_{i_{k}}\right):=b_{e_{i_{1}}+\cdots+e_{i_{k}}}$, where

$$
b(x):=\sum_{\substack{r \in \mathbb{Z}_{+}^{n} \\|r|=k}} b_{r} \frac{x^{r}}{r !}
$$

and extend $T_{k}(b)$ multilinearly. Hence, $T_{k}(b)$ is multilinear and symmetric.

On p. 255 of [6], for $c \in \mathbb{R}^{n}$ and $k \geq 1$, the mapping $\sigma_{c}: S_{k}^{l} \rightarrow S_{k-1}^{l}$ was defined by

$$
\sigma_{c}(b)(x):=\sum_{\substack{s \in \mathbb{Z}_{+}^{n} \\|s|=k-1}}\left(\sum_{i=1}^{n} b_{s+e_{i}} c_{i}\right) \frac{x^{s}}{s !} \quad \text { for all } \quad x \in U(h),
$$

where, as before, $b(x)=\sum_{r \in \mathbb{Z}_{+}^{n},|r|=k} b_{r}\left(x^{r} / r !\right)$. We define

$$
\tilde{\omega}:=\sigma_{\left(h_{1}, \ldots, h_{n}\right)} .
$$

Proposition 5 and the proof of Theorem 20 of [6], yield that the volume $V$ is a linear $d$-stress. Hence, $\tilde{\omega}^{d-k}(V) \in S_{k}^{l}$ for $k=0, \ldots, d$ (see Theorem 8 of [6]).

The aim of this section is to prove the following theorem.

Theorem 6. Let $k \in\{2, \ldots, d\}$, let $c \in \mathbb{R}^{n}$. The equation

$$
T_{k}\left(\tilde{\omega}^{d-k}(V)\right)\left(e_{i_{1}}, \ldots, e_{i_{k-1}}, c\right)=0
$$

holds for all $\left\{i_{1}, \ldots, i_{k-1}\right\} \in \Delta_{k-1}$ if and only if $c$ is the support vector of a point.

The proof of Theorem 6 requires some additional results which we derive first. For given $b \in S_{k}^{l}$, we define by

$$
\Phi_{k}^{b}(x):=\sigma_{x}(b)
$$

a linear mapping $\Phi_{k}^{b}: \mathbb{R}^{n} \rightarrow S_{k-1}^{l}$. The kernel ker $\Phi_{k}^{b}$ of this mapping can be described as follows.

Proposition 7. Let $k \in\{2, \ldots, d\}$ and $c \in \mathbb{R}^{n}$. Then $c \in \operatorname{ker} \Phi_{k}^{b}$ if and only if

$$
T_{k}(b)\left(e_{i_{1}}, \ldots, e_{i_{k-1}}, c\right)=0
$$

for all $i_{1}, \ldots, i_{k-1} \in\{1, \ldots, n\}$.

Proof. Let $c=\left(c_{1}, \ldots, c_{n}\right) \in \operatorname{ker} \Phi_{k}^{b}$, where $b(x)=\sum_{r \in \mathbb{Z}_{+}^{n},|r|=k} b_{r}\left(x^{r} / r !\right)$. Because of (10), this is equivalent to

$$
\sum_{i=1}^{n} b_{s+e_{i}} c_{i}=0 \quad \text { for all } \quad s \in \mathbb{Z}_{+}^{n} \quad \text { with } \quad|s|=k-1 .
$$

Let $i_{1}, \ldots, i_{k-1} \in\{1, \ldots, n\}$ and define $s:=e_{i_{1}}+\cdots+e_{i_{k-1}}$. Then $s \in \mathbb{Z}_{+}^{n}$ and $|s|=k-1$. Conversely, if $s \in \mathbb{Z}_{+}^{n}$ and $|s|=k-1$, then $s=e_{i_{1}}+\cdots+e_{i_{k-1}}$ with suitable $i_{1}, \ldots, i_{k-1} \in\{1, \ldots, n\}$. 
In both cases, we have

$$
\begin{aligned}
T_{k}(b)\left(e_{i_{1}}, \ldots, e_{i_{k-1}}, c\right) & =\sum_{i=1}^{n} T_{k}(b)\left(e_{i_{1}}, \ldots, e_{i_{k-1}}, e_{i}\right) c_{i} \\
& =\sum_{i=1}^{n} b_{e_{i_{1}}+\cdots+e_{i_{k-1}}+e_{i}} c_{i} \\
& =\sum_{i=1}^{n} b_{s+e_{i}} c_{i} .
\end{aligned}
$$

The proposition now follows from (13).

By Lemma 5.1.2 of [8], the volume function $V$ can be represented as

$$
V(x)=\sum_{j_{1}=1}^{n} \cdots \sum_{j_{d}=1}^{n} a_{j_{1} \cdots j_{d}} x_{j_{1}} \cdots x_{j_{d}}
$$

where the coefficients $a_{j_{1} \ldots j_{d}} \in \mathbb{R}$ are symmetric in $j_{1}, \ldots, j_{d}$. For given $r=\left(r_{1}, \ldots\right.$, $\left.r_{n}\right) \in \mathbb{Z}_{+}^{n}$ with $|r|=d$, there are pairwise disjoint numbers $i_{1}, \ldots, i_{m} \in\{1, \ldots, n\}$ such that $r_{i_{s}}>0$ for all $s=1, \ldots, m$ and $r=r_{i_{1}} e_{i_{1}}+\cdots+r_{i_{m}} e_{i_{m}}$. Hence, $\left\{i_{1}, \ldots, i_{m}\right\}=$ $\operatorname{supp} x^{r}$. We then define

$$
a_{r}:=a_{r_{i_{1}}}^{a_{i_{1} \cdots i_{1}} \cdots} \underbrace{i_{m} \cdots i_{m}}_{r_{i_{m}}} \text {. }
$$

This is well defined because the coefficients are symmetric. For $r=\left(r_{1}, \ldots, r_{n}\right) \in \mathbb{Z}_{+}^{n}$ with $|r|=k$ we then obtain

$$
\frac{\partial^{k} V}{\partial x^{r}}(x)=d ! \sum_{\substack{s \in \mathbb{Z}_{+}^{n} \\|s|=d-k}} a_{r+s} \frac{x^{s}}{s !},
$$

where we defined

$$
\frac{\partial^{k} V}{\partial x^{r}}(x):=\frac{\partial^{k} V}{\partial x_{n}^{r_{n}} \cdots \partial x_{1}^{r_{1}}}(x)
$$

for $r=\left(r_{1}, \ldots, r_{n}\right) \in \mathbb{Z}_{+}^{n}$ with $|r|=k$.

Next, we define

$$
W_{k}(x):=\sum_{\substack{r \in \mathbb{Z}_{+}^{n} \\|r|=k}} \frac{\partial^{k} V}{\partial x^{r}}(h) \frac{x^{r}}{r !} \quad \text { for } \quad x \in U(h),
$$

$k \in\{0, \ldots, d\}$. In particular,

$$
W_{d}(x)=d ! \sum_{\substack{r \in \mathbb{Z}_{+}^{n} \\|r|=d}} a_{r} \frac{x^{r}}{r !}=V(x) .
$$

The following lemma shows that $\tilde{\omega}^{d-k}(V)$ and $W_{k}$ are equal up to the factor $(d-k)$ ! (see Theorem 21 of [6]). 
Lemma 8. Let $k \in\{0, \ldots, d\}$. Then $\tilde{\omega}^{d-k}(V)=(d-k) ! W_{k}$.

Proof. We have just seen that $\tilde{\omega}^{0}(V)=V=W_{d}$. Let $k \in\{0, \ldots, d-1\}$ and assume that $\tilde{\omega}^{k}(V)=k ! W_{d-k}$. Then

$$
\begin{aligned}
\tilde{\omega}^{k+1}(V)(x) & =\tilde{\omega}\left(\tilde{\omega}^{k}(V)\right)(x) \\
& =\tilde{\omega}\left(k ! W_{d-k}\right)(x) \\
& =k ! \sum_{\substack{r \in \mathbb{Z}_{+}^{n} \\
|r|=d-(k+1)}}\left(\sum_{i=1}^{n} \frac{\partial^{d-k} V}{\partial x^{r+e_{i}}}(h) h_{i}\right) \frac{x^{r}}{r !} \\
& =\left.k ! \sum_{\substack{r \in \mathbb{Z}_{+}^{n} \\
|r|=d-(k+1)}} \frac{d}{d t}\right|_{t=0} \frac{\partial^{d-(k+1)} V}{\partial x^{r}}(h+t h) \frac{x^{r}}{r !} \\
& =\left.k ! \sum_{\substack{r \in \mathbb{Z}_{+}^{n} \\
|r|=d-(k+1)}} \frac{d}{d t}\right|_{t=0}(1+t)^{k+1} \frac{\partial^{d-(k+1)} V}{\partial x^{r}}(h) \frac{x^{r}}{r !} \\
& =(k+1) ! \sum_{\substack{r \in \mathbb{Z}_{+}^{n} \\
|r|=d-(k+1)}} \frac{\partial^{d-(k+1)} V}{\partial x^{r}}(h) \frac{x^{r}}{r !} \\
& =(k+1) ! W_{d-(k+1)}(x)
\end{aligned}
$$

for all $x \in U(h)$. This proves the assertion.

The proof of Theorem 10 requires the following lemma. In this lemma we denote by

$$
L\left(u_{1}, \ldots, u_{n}\right):=\left\{\left(\alpha_{1}, \ldots, \alpha_{n}\right) \in \mathbb{R}^{n} \mid \sum_{i=1}^{n} \alpha_{i} u_{i}=o\right\}
$$

the set of linear dependences of $u_{1}, \ldots, u_{n}$. This is a linear subspace of dimension $n-d$.

Lemma 9. Let $k \in\{2, \ldots, d\}$ and

$$
\tilde{\omega}^{d-k}(V)(x)=\sum_{\substack{r \in \mathbb{Z}_{+}^{n} \\|r|=k}} b_{r} \frac{x^{r}}{r !}
$$

Then

$$
\operatorname{lin}\left\{\left(b_{s+e_{1}}, \ldots, b_{s+e_{n}}\right)\left|s \in \mathbb{Z}_{+}^{n},\right| s \mid=k-1\right\}=L\left(u_{1}, \ldots, u_{n}\right) .
$$


Proof. The definition of a $k$-stress includes that

$$
\left(b_{s+e_{1}}, \ldots, b_{s+e_{n}}\right) \in L\left(u_{1}, \ldots, u_{n}\right)
$$

for all $s \in \mathbb{Z}_{+}^{n}$ with $|s|=k-1$ (see (9)). Hence,

$$
\operatorname{lin}\left\{\left(b_{s+e_{1}}, \ldots, b_{s+e_{n}}\right)\left|s \in \mathbb{Z}_{+}^{n},\right| s \mid=k-1\right\} \subset L\left(u_{1}, \ldots, u_{n}\right) .
$$

The proof of (15) is completed by induction on $k$. Inserting $k=2$ into (14) we obtain

$$
W_{2}(x)=\sum_{\substack{r \in \mathbb{Z}_{+}^{n} \\|r|=2}} \frac{\partial^{2} V}{\partial x^{r}}(h) \frac{x^{r}}{r !} .
$$

Using Lemma 8 we see that

$$
b_{e_{i}+e_{j}}=(d-2) ! \frac{\partial^{2} V}{\partial x_{j} \partial x_{i}}(h) .
$$

For $\{i, j\} \in \Delta_{2}$, Theorem 4 then yields

$$
\begin{aligned}
b_{e_{i}+e_{j}} & =(d-2) !\left[u_{i}, u_{j}\right]^{-1} V_{d-2}\left(F_{i} \cap F_{j}\right) \\
& =(d-2) ! \operatorname{cosec} \theta_{i j} V_{d-2}\left(F_{i} \cap F_{j}\right) .
\end{aligned}
$$

In this formula, $\theta_{i j}$ denotes the angle between $u_{i}$ and $u_{j}$. If $\{i, j\} \notin \Delta_{2}$ and $i \neq j$, then Proposition 5 implies that

$$
b_{e_{i}+e_{j}}=(d-2) ! \frac{\partial^{2} V}{\partial x_{j} \partial x_{i}}(h)=0 .
$$

For $i \in\{1, \ldots, n\}$, we have $\sum_{j=1}^{n} b_{e_{i}+e_{j}} u_{j}=o$ using (9). Hence,

$$
\begin{aligned}
b_{2 e_{i}} & =-\sum_{j \neq i} b_{e_{i}+e_{j}}\left\langle u_{i}, u_{j}\right\rangle \\
& =-(d-2) ! \sum_{\{i, j\} \in \Delta_{2}} \cot \theta_{i j} V_{d-2}\left(F_{i} \cap F_{j}\right) .
\end{aligned}
$$

Summarizing (17), (18), and (19) we obtain

$$
b_{e_{i}+e_{j}}= \begin{cases}(d-2) ! \operatorname{cosec} \theta_{i j} V_{d-2}\left(F_{i} \cap F_{j}\right) & \text { for }\{i, j\} \in \Delta_{2}, \\ 0 & \text { for }\{i, j\} \notin \Delta_{2}, \quad i \neq j, \\ -(d-2) ! \sum_{\{i, m\} \in \Delta_{2}} \cot \theta_{i m} V_{d-2}\left(F_{i} \cap F_{m}\right) & \text { for } i=j .\end{cases}
$$

We now consider the $n \times n$ matrix $\left(b_{e_{i}+e_{j}}\right)_{i, j=1, \ldots, n}$. Using the above representation of $b_{e_{i}+e_{j}}$ we see that

$$
\begin{aligned}
\left(W_{1}, \ldots, W_{n}\right)^{t} \in \operatorname{ker}\left(b_{e_{i}+e_{j}}\right)_{i, j=1, \ldots, n} & \\
& \Leftrightarrow \sum_{j=1}^{n} b_{e_{i}+e_{j}} W_{j}=0 \quad \text { for all } i=1, \ldots, n \\
& \Leftrightarrow \sum_{\{i, j\} \in \Delta_{2}} b_{e_{i}+e_{j}} W_{j}+b_{2 e_{i}} W_{i}=0 \quad \text { for all } i=1, \ldots, n \\
& \Leftrightarrow \sum_{\{i, j\} \in \Delta_{2}} W_{i j} V_{d-2}\left(F_{i} \cap F_{j}\right)=0 \quad \text { for all } i=1, \ldots, n,
\end{aligned}
$$


where $W_{i j}$ is defined by

$$
W_{i j}:=W_{j} \operatorname{cosec} \theta_{i j}-W_{i} \cot \theta_{i j} .
$$

Theorem 4.1.1 in [2] shows that (20) implies

$$
\operatorname{rank}\left(b_{e_{i}+e_{j}}\right)_{i, j=1, \ldots, n}=n-d
$$

(see also p. 11 of [4]). Thus, by (16) and because of

$$
\operatorname{dim} L\left(u_{1}, \ldots, u_{n}\right)=n-d,
$$

the assertion is true if $k=2$.

Let $k \in\{2, \ldots, d-1\}$ and assume that the assertion is proved for $\tilde{\omega}^{d-k}(V)$. Let

$$
\tilde{\omega}^{d-k}(V)(x)=\sum_{\substack{r \in \mathbb{Z}_{+}^{n} \\|r|=k}} b_{r} \frac{x^{r}}{r !}
$$

and

$$
\tilde{\omega}^{d-(k+1)}(V)(x)=\sum_{\substack{r \in \mathbb{Z}_{+}^{n} \\|r|=k+1}} c_{r} \frac{x^{r}}{r !} .
$$

Because of $\tilde{\omega}^{d-k}(V)=\tilde{\omega}\left(\tilde{\omega}^{d-(k+1)}(V)\right)$ we have

$$
b_{r}=\sum_{i=1}^{n} c_{r+e_{i}} h_{i} \quad \text { for all } \quad r \in \mathbb{Z}_{+}^{n} \text { with }|r|=k .
$$

The assumption of induction yields

$$
\operatorname{lin}\left\{\left(b_{s+e_{1}}, \ldots, b_{s+e_{n}}\right)\left|s \in \mathbb{Z}_{+}^{n},\right| s \mid=k-1\right\}=L\left(u_{1}, \ldots, u_{n}\right) .
$$

Furthermore, since $o \in$ int $P$, we have $h_{1}, \ldots, h_{n}>0$ and thus

$$
\begin{aligned}
& \operatorname{dim} \operatorname{lin}\left\{\left(c_{s+e_{i}+e_{1}} h_{1}, \ldots, c_{s+e_{i}+e_{n}} h_{n}\right)\left|s \in \mathbb{Z}_{+}^{n},\right| s \mid=k-1, i=1, \ldots, n\right\} \\
& =\operatorname{dim} \operatorname{lin}\left\{\left(c_{s+e_{i}+e_{1}}, \ldots, c_{s+e_{i}+e_{n}}\right)\left|s \in \mathbb{Z}_{+}^{n},\right| s \mid=k-1, i=1, \ldots, n\right\} .
\end{aligned}
$$

We also know that for each $r \in \mathbb{Z}_{+}^{n}$ with $|r|=k$ there are $s \in \mathbb{Z}_{+}^{n},|s|=k-1$, and $i \in\{1, \ldots, n\}$ such that $r=s+e_{i}$. Combining these observations we deduce that

$$
\begin{aligned}
& \operatorname{dim} L\left(u_{1}, \ldots, u_{n}\right) \\
& \stackrel{(22)}{=} \operatorname{dim} \operatorname{lin}\left\{\left(b_{s+e_{1}}, \ldots, b_{s+e_{n}}\right)\left|s \in \mathbb{Z}_{+}^{n},\right| s \mid=k-1\right\} \\
& \stackrel{(21)}{=} \operatorname{dim} \operatorname{lin}\left\{\sum_{i=1}^{n}\left(c_{s+e_{i}+e_{1}} h_{1}, \ldots, c_{s+e_{i}+e_{n}} h_{n}\right)\left|s \in \mathbb{Z}_{+}^{n},\right| s \mid=k-1\right\} \\
& \leq \operatorname{dim} \operatorname{lin}\left\{\left(c_{s+e_{i}+e_{1}} h_{1}, \ldots, c_{s+e_{i}+e_{n}} h_{n}\right)\left|s \in \mathbb{Z}_{+}^{n},\right| s \mid=k-1, i=1, \ldots, n\right\} \\
& \stackrel{(23)}{=} \operatorname{dim} \operatorname{lin}\left\{\left(c_{s+e_{i}+e_{1}}, \ldots, c_{s+e_{i}+e_{n}}\right)\left|s \in \mathbb{Z}_{+}^{n},\right| s \mid=k-1, i=1, \ldots, n\right\} \\
&=\operatorname{dim} \operatorname{lin}\left\{\left(c_{r+e_{1}}, \ldots, c_{r+e_{n}}\right)\left|r \in \mathbb{Z}_{+}^{n},\right| r \mid=k\right\} \\
& \stackrel{(16)}{\leq} \operatorname{dim} L\left(u_{1}, \ldots, u_{n}\right),
\end{aligned}
$$


that is, the linear subspace

$$
\operatorname{lin}\left\{\left(c_{r+e_{1}}, \ldots, c_{r+e_{n}}\right)\left|r \in \mathbb{Z}_{+}^{n},\right| r \mid=k\right\}
$$

has the same dimension as $L\left(u_{1}, \ldots, u_{n}\right)$. By (16), this is only possible if

$$
\operatorname{lin}\left\{\left(c_{r+e_{1}}, \ldots, c_{r+e_{n}}\right)\left|r \in \mathbb{Z}_{+}^{n},\right| r \mid=k\right\}=L\left(u_{1}, \ldots, u_{n}\right) .
$$

Hence, (15) holds true.

We now define $\Phi_{k}:=\Phi_{k}^{\tilde{\omega}^{d-k}(V)}$. Using Lemma 9 we are able to determine the kernel of $\Phi_{k}$.

Theorem 10. Let $k \in\{2, \ldots, d\}$. Then

$$
\operatorname{ker} \Phi_{k}=\left\{\left(\left\langle a, u_{1}\right\rangle, \ldots,\left\langle a, u_{n}\right\rangle\right)=\mid a \in \mathbb{R}^{d}\right\} .
$$

Proof. Let

$$
\tilde{\omega}^{d-k}(V)(x)=\sum_{\substack{r \in \mathbb{Z}_{+}^{n} \\|r|=k}} b_{r} \frac{x^{r}}{r !} .
$$

Then $c=\left(c_{1}, \ldots, c_{n}\right) \in \operatorname{ker} \Phi_{k}$ holds if and only if $\sum_{i=1}^{n} b_{s+e_{i}} c_{i}=0$ for all $s \in \mathbb{Z}_{+}^{n}$ with $|s|=k-1$. Lemma 9 shows that this is equivalent to $c \in L\left(u_{1}, \ldots, u_{n}\right)^{\perp}$. We have $L\left(u_{1}, \ldots, u_{n}\right)=\operatorname{ker} A$, where $A$ is the $d \times n$ matrix whose $i$ th column is the vector $u_{i}$, that is, $A:=\left(u_{1}, \ldots, u_{n}\right)$. Hence,

$$
\begin{aligned}
\operatorname{ker} \Phi_{k} & =L\left(u_{1}, \ldots, u_{n}\right)^{\perp} \\
& =(\operatorname{ker} A)^{\perp} \\
& =\operatorname{im} A^{t} \\
& =\left\{\left(\left\langle a, u_{1}\right\rangle, \ldots,\left\langle a, u_{n}\right\rangle\right) \mid a \in \mathbb{R}^{d}\right\} .
\end{aligned}
$$

Here, im $A^{t}$ denotes the image of the transpose $A^{t}$ of $A$.

We are now in a position to prove Theorem 6.

Proof of Theorem 6. It is an immediate consequence of Proposition 7 and Theorem 10 that (11) holds for all $i_{1}, \ldots, i_{k-1} \in\{1, \ldots, n\}$ if and only if $c \in \mathbb{R}^{n}$ is the support vector of a point. Hence, we only have to prove that (11) for all $\left\{i_{1}, \ldots, i_{k-1}\right\} \in \Delta_{k-1}$ implies that this equation holds for all $i_{1}, \ldots, i_{k-1} \in\{1, \ldots, n\}$.

Assume that (11) holds for all $\left\{i_{1}, \ldots, i_{k-1}\right\} \in \Delta_{k-1}$. First let $i_{1}, \ldots, i_{k-1} \in\{1, \ldots, n\}$ be pairwise disjoint. If $\left\{i_{1}, \ldots, i_{k-1}\right\} \notin \Delta_{k-1}$, then $\left\{i_{1}, \ldots, i_{k-1}, i\right\} \notin \Delta$ for all $i \in$ $\{1, \ldots, n\}$. Hence,

$$
T_{k}\left(\tilde{\omega}^{d-k}(V)\right)\left(e_{i_{1}}, \ldots, e_{i_{k-1}}, e_{i}\right)=0
$$

for all $i=1, \ldots, n$ because of $\tilde{\omega}^{d-k}(V) \in S_{k}^{l}$ (see (8)). We obtain

$$
T_{k}\left(\tilde{\omega}^{d-k}(V)\right)\left(e_{i_{1}}, \ldots, e_{i_{k-1}}, c\right)=0 .
$$


We now drop the assumption of pairwise disjointness and choose arbitrary numbers $i_{1}, \ldots, i_{k-1} \in\{1, \ldots, n\}$. We define

$$
r:=e_{i_{1}}+\cdots+e_{i_{k-1}} .
$$

Let $s \in \mathbb{Z}_{+}^{n},|s|=k-1$. Then $s$ has a unique representation $s=e_{j_{1}}+\cdots+e_{j_{k-1}}$ with $j_{1}, \ldots, j_{k-1} \in\{1, \ldots, n\}$. For $c=\left(c_{1}, \ldots, c_{n}\right) \in \mathbb{R}^{n}$ define

$$
T_{k}(s, c):=T_{k}\left(\tilde{\omega}^{d-k}(V)\right)\left(e_{j_{1}}, \ldots, e_{j_{k-1}}, c\right) .
$$

We then have

$$
\sum_{\substack{s \in \mathbb{Z}_{+}^{n} \\|s|=k-1}} T_{k}(s, c) \frac{x^{s}}{s !}=\sigma_{c}\left(\tilde{\omega}^{d-k}(V)\right)(x) \quad \text { for all } \quad x \in U(h) .
$$

This shows that the mapping

$$
x \mapsto \sum_{\substack{s \in \mathbb{Z}_{+}^{n} \\|s|=k-1}} T_{k}(s, c) \frac{x^{s}}{s !}
$$

is a linear $(k-1)$-stress.

Since $P$ is a simple polytope, for $m \in\{1, \ldots, d\}$ and $\left\{i_{1}, \ldots, i_{m}\right\} \in \Delta_{m}$, the vectors $u_{i_{1}}, \ldots, u_{i_{m}}$ are linearly independent. Thus, $u_{1}, \ldots, u_{n}$ are in general relative position with respect to $\Delta$ in the sense of Lee [6, p. 258]. Together with the fact that (27) is a linear $(k-1)$-stress, the general relative position of the outer normal vectors as well as Theorem 12 of [6], and (25), imply that

$$
T_{k}(r, c)=\sum_{\left\{j_{1}, \ldots, j_{k-1}\right\} \in \Delta_{k-1}} \alpha_{j_{1}, \ldots, j_{k-1}}^{(r, c)} T_{k}\left(\tilde{\omega}^{d-k}(V)\right)\left(e_{j_{1}}, \ldots, e_{j_{k-1}}, c\right),
$$

where $\alpha_{j_{1}, \ldots, j_{k-1}}^{(r, c)} \in \mathbb{R}$ and $r$ is defined by (26).

The assumption that (11) holds for all $\left\{j_{1}, \ldots, j_{k-1}\right\} \in \Delta_{k-1}$ now implies

$$
T_{k}\left(\tilde{\omega}^{d-k}(V)\right)\left(e_{i_{1}}, \ldots, e_{i_{k-1}}, c\right)=T_{k}(r, c)=0 .
$$

Hence, (11) holds for all $i_{1}, \ldots, i_{k-1} \in\{1, \ldots, n\}$.

\section{Proof of Theorem 1}

After these preparations it is not difficult to prove the extension of Aleksandrov's result on the infinitesimal rigidity of convex $d$-dimensional polytopes with stationary facet volumes (see Theorem 1 on p. 407 of [1]).

Proof of Theorem 1. Without loss of generality we may assume that $o \in \operatorname{int} P$. Let $F \in \mathcal{F}_{k}(P)$. Since $P$ is a simple polytope, the face $F$ is the intersection of exactly $d-k$ facets of $P$. Let $u_{i_{1}}, \ldots, u_{i_{d-k}}$ be the outer normal vectors of these facets. 
We have

$$
\begin{aligned}
\left.\frac{d}{d t}\right|_{t=0} V_{k}(F(h+t c)) & =\left.\left[u_{i_{1}}, \ldots, u_{i_{d-k}}\right] \frac{d}{d t}\right|_{t=0} \frac{\partial^{d-k} V}{\partial x_{i_{d-k}} \cdots \partial x_{i_{1}}}(h+t c) \\
& =\left[u_{i_{1}}, \ldots, u_{i_{d-k}}\right] \sum_{i=1}^{n} c_{i} \frac{\partial^{d-k+1} V}{\partial x_{i} x_{i_{d-k}} \cdots \partial x_{i_{1}}}(h) \\
& =\frac{\left[u_{i_{1}}, \ldots, u_{i_{d-k}}\right]}{(k-1) !} T_{d-k+1}\left(\tilde{\omega}^{k-1}(V)\right)\left(e_{i_{1}}, \ldots, e_{i_{d-k}}, c\right),
\end{aligned}
$$

where the first step follows because of Theorem 4 and where we have applied Lemma 8 in the final step. Thus, (3) is equivalent to

$$
T_{d-k+1}\left(\tilde{\omega}^{k-1}(V)\right)\left(e_{i_{1}}, \ldots, e_{i_{d-k}}, c\right)=0
$$

for all $\left\{i_{1}, \ldots, i_{d-k}\right\} \in \Delta_{d-k}$. We have $d-k+1 \in\{2, \ldots, d\}$, because $k \in\{1, \ldots, d-1\}$. Theorem 6 implies that $c$ is the support vector of a point.

\section{References}

1. A. D. Aleksandrov: Konvexe Polyeder. Akademie-Verlag, Berlin, 1958.

2. Ch. Bauer: Polytope in der Brunn-Minkowski-Theorie. Thesis, Universität Freiburg, 1996.

3. A. Brøndsted: An Introduction to Convex Polytopes. Springer-Verlag, Heidelberg, 1983.

4. P. Filliman: Rigidity and the Alexandrov-Fenchel inequality. Monatsh. Math. 113 (1992), 1-22.

5. C. W. Lee: Some recent results on convex polytopes. Contemp. Math. 114 (1990), 3-19.

6. C. W. Lee: Generalized stress and motion. In: Polytopes: Abstract, Convex and Computational (Scarborough, 1993; eds. T. Bisztriczky et al.). NATO ASI Series C, vol. 440, Kluwer, Dordrecht, 1994, pp. 249271.

7. C. W. Lee: P.L.-Spheres, convex polytopes, and stress. Discrete Comput. Geom. 15 (1996), 389-421.

8. R. Schneider: Convex Bodies: The Brunn-Minkowski Theory. Encyclopedia of Mathematics and its Applications., vol. 44. Cambridge University Press, Cambridge, 1993.

Received November 20, 1997. 